\title{
Atomistics of superlubricity
}

\author{
Motohisa HIRANO* \\ Department of Mechanical Engineering, Faculty of Science and Engineering; Hosei University 3-7-2, Kajino, Koganei, 184-8584 Tokyo \\ Received: 23 February 2014 / Revised: 19 April 2014 / Accepted: 22 April 2014 \\ (C) The author(s) 2014. This article is published with open access at Springerlink.com
}

\begin{abstract}
Friction is a phenomenon observed ubiquitously in daily life, yet its nature is complicated. Friction between rough surfaces is considered to arise primarily because of macroscopic roughness. In contrast, interatomic forces dominate between clean and smooth surfaces. "Superlubricity", where friction effectively becomes zero, occurs when the ratio of lattice parameters in the pair of surfaces becomes an irrational number. Superlubricity has been found to exist in a limited number of systems, but is a very important phenomenon both in industry and in mechanical engineering. New atomistic research on friction is under way, with the aim of refining theoretical models that consider interactions between atoms beyond mean field theory and experiments using ultrahigh vacuum non-contact atomic force microscopy. Such research is expected to help clarify the nature of microscopic friction, reveal the onset conditions of friction and superlubricity as well as the stability of superlubricity, discover new superlubric systems, and lead to new applications.
\end{abstract}

Keywords: atomic-level friction; atomistics of friction; nanotribology; superlubricity

\section{Friction and superlubricity}

Superlubricity, which is the state of vanishing friction, is closely related to the origin of friction. The following is an overview of the history of research on the origin of friction, including the molecular theory of friction and the problem of energy dissipation in friction.

Sota wrote an interesting article on the history of friction research science [1]. The science of friction started in Italy during the renaissance period in the $15^{\text {th }}$ century. Leonardo da Vinci carefully observed and experimented on stones and wood found in daily life and introduced the concept of the friction coefficient. More than 200 years passed without any description in friction research, until much discussion of the laws regarding friction and the origin of friction started to happen in the $17^{\text {th }}$ to $18^{\text {th }}$ century. The results of research were applied to engineering in the form of lubrication technology during the industrial revolution in the $18^{\text {th }}$ century, and research by Coulomb and others were summarized as "basic laws of friction".

* Corresponding author: Motohisa HIRANO.

E-mail: hirano@hosei.ac.jp
The principles of how friction happens were discussed from the end of the $17^{\text {th }}$ century to around the middle of the $18^{\text {th }}$ century as mentioned above, and Coulomb completed his roughness theory. In contrast, Desaguliers proposed the molecular theory, which is the complete opposite of the roughness theory, around the middle of the $18^{\text {th }}$ century. The molecular theory considers the true cause of friction to be the interaction of molecular forces at the contacting surfaces where friction happens. In other words, this theory states that a smoother surface means that the friction surfaces come together, increasing the interference between surface forces stemming from interatomic interactions. Desaguliers extracted a few millimeter-sized pieces from a lead sphere, and found that strongly pressing such pieces against each other resulted in strong bonding between the pieces. Further observation of the remains after separation showed that only a fraction of the pressed surface had actually been in contact. This finding gave rise to the prediction that "friction ultimately increases if surfaces are continuously polished smoothly". This prediction was proved by Hardy's experiment in the $20^{\text {th }}$ century with improvements in 
surface processing technologies [2]. He carried out experiments on the friction of glass surfaces and showed that glass surfaces that are polished very carefully such as those in lenses have greater friction than glass with rough surfaces. He also found that tracks of wear caused by friction are initially about 1 micron wide, and as friction gradually increases wear, the width increases to about 50 microns. This experiment refuted the roughness theory and proved that friction is not only a problem of energy loss from the interaction of molecular forces but also is a phenomenon of plastic deformation and fracture in materials science that accompanies destruction of the surface. Holm's experiment demonstrated that the friction between clean surfaces is high under high vacuum and that minute amounts of gas molecule adsorption significantly decrease friction [3]. Thus, molecular theory gained evidence and became the foundation of the atomistics of friction. On the other hand, regarding the friction model of actual surfaces, the contact model was refined through Holm's true contact concept and Mises's yield theory. Relations between friction forces and materials properties were investigated in detail in terms of adhesion theory based on shear models of the adhesion range at the true contact area [4]. The findings resulted in today's lubrication technologies for head-disk interfaces in contact start-stop type magnetic disc devices, and lubrication technologies will become even more important in head-disk constant contact type devices in the future.

\section{Friction and atomistics}

The work from friction has very different properties from the work from gravity. Work from gravity happens when objects are moved against gravity, which is always acting on objects. In contrast, friction is the force required to move objects perpendicular to the direction of gravity. Once sliding motion starts, friction appears as resistance against the sliding motion and results in work from friction. Therefore, friction has the interesting property that it appears when objects start sliding and disappears when objects stop. Even in interatomic forces, no work from friction is generated as long as the combined interatomic force is perpendicular to the sliding direction. The British physicist
Tomlinson [5] was the first to explain this finding at the start of the $20^{\text {th }}$ century, at around the same time as the British chemist Dalton established the modern atomistics.

The modern atomistics was established after physics reached the level of atoms in the $19^{\text {th }}$ century. Physics started to consider atoms around the mid- $19^{\text {th }}$ century although the original concept of atomistics itself, which is that matter consists of atoms, is thought to have emerged in ancient Greece as "particle philosophy". The British physicist-chemist Boyle tried to use "particle philosophy" as the foundation of chemistry, and his attempt to build chemistry upon particle philosophy materialized in the early $19^{\text {th }}$ century as Dalton's atomistics. Dalton postulated that objects with size that are touched daily, regardless of whether the objects are in gas, liquid, or solid form, consist of a vast number of very minute particles or atoms bound together by interatomic force. He thought that there is attraction and repulsion between atoms and that the balance between these opposing forces results in the three states of gas, liquid, and solid. The attraction and repulsion between atoms was later explained based on the concept of electron energy levels and electron states in quantum mechanics. Dalton's atomistics was improved through corrections by Avogadro and others. Although there were opponents to atomistics, it explained many experimental findings about the materials properties of gases, Boyle's law, diffusion and viscosity of gases, laws on heat conductivity, and the law of increasing entropy. Atomistics later provided an important foundation for problems regarding the nature of heat. Physicists such as Helmholtz came to believe that atoms govern thermal motion.

Tomlinson's paper states early on that "friction is generally recognized to happen because of interactions between molecules that are very close to each other" [5]. He investigated the forces that appear in relative motion of atoms in the field of interatomic interactions at the friction surface, and succeeded in rationally explaining the problem of how friction arises from interatomic interactions at the contact surface, or how mechanical energy dissipates into heat energy due to friction, by introducing the concept of "adiabaticity", thereby opening the door to the atomistic theory of friction. Figure 1 shows the original model in the paper. 


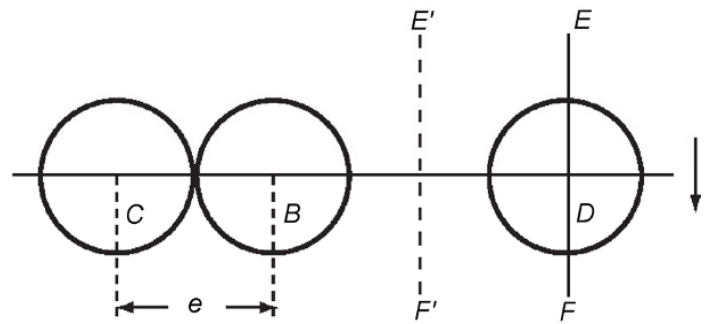

Fig. 1 Tomlinson's single-pair atom system for explaining energy dissipation in friction.

However, very little research on the atomistics of friction followed because of the difficulty of handling the complexity of actual surfaces based on the theory.

Friction research changed completely with recent advances in nanotechnology. Friction research in ideal systems where many factors of friction are identified has been difficult for experimental technology reasons; however, recent measurement technologies, including scanning probe microscopy and technologies to control clean surfaces under ultrahigh vacuum, have enabled direct comparison between theoretical models and experiments $[6,7]$. Theory can investigate in detail the fundamental properties of interatomic interactions and the mechanism of friction generation using computational experiments on atomistic models [8]. Therefore, "ideal friction experiments", where the causes of friction are accurately identified, can be combined with "atomic scale friction simulations", and thus the adequacy of atomic scale friction theory can now be directly verified. For example, atomic force microscopy can accurately measure the friction between the surface of a needle tip attached to the end of a cantilever and the surface of a sample using the optical lever method, which is a displacement measurement method. The latest experimental devices have enabled the first observations of friction without wear or fracture [9]. Conventional adhesion theory cannot be used to investigate such friction without wear, and therefore it was necessary to clarify the origins of friction in terms of atomistics [10].

\section{Atomistic models}

Uncovering the principles of energy dissipation in friction has been recognized as an important problem for a long time. For friction phenomena caused by adsorption at the true contact area, which has been observed the most, friction energy has been considered to dissipate from plastic deformation at the true contact area $[3,4]$. This is the basic concept of adhesion theory, which postulates that bumps on the surface dig into the other surface and cause wear particle because of plastic deformation and fracture, and the accumulation of such behavior results in energy dissipation. The principle is the same as the idea that the energy necessary for the deformation of bulk materials at the macroscopic scale is due to the dissipation by motion of dislocations and propagation of cracks in the material. However, friction experiments at the atomic scale mentioned in the previous section revealed new friction phenomena that do not accompany plastic deformation or wear, and thus the problem of energy dissipation in friction regained attention in relation to atomistics.

McClelland [10] built an atomistic model in which infinite planes slide against each other to investigate the problem of energy dissipation in a wear-free friction model (Fig. 2). Atoms positioned above do not interact with each other in the independent oscillator model in Fig. 2(b), and so the model is essentially the same as Tomlinson's model. Figure 2(c) is the FrenkelKontorova model described later. This model assumes that strong forces such as metallic or covalent bonds act on atoms in solids, and relatively weak forces such as van der Waals forces or hydrogen bonds act between atoms at the surfaces above and below. The idea behind this model is that in the charge density wave model, which is a physical system that describes sliding motion similar to friction, it is well known that charge density waves from interactions between ionic crystals during sliding result in unstable sliding when the interactions become even slightly strong, as mentioned below. In friction models with such properties, when the surfaces in contact are incommensurate, or when the ratio of periodicity along sliding surfaces above and below is an irrational number, the two surfaces are found to be able to slide without energy dissipation. Such sliding phenomenon without energy dissipation may be unfamiliar in the field of tribology, but is well known to appear ubiquitously in some physical systems with two interacting periodicities [11]. Examples of such physical systems include charge density waves, ionic conductivity, epitaxial crystal growth, and adhesive atom layers. 
(a)

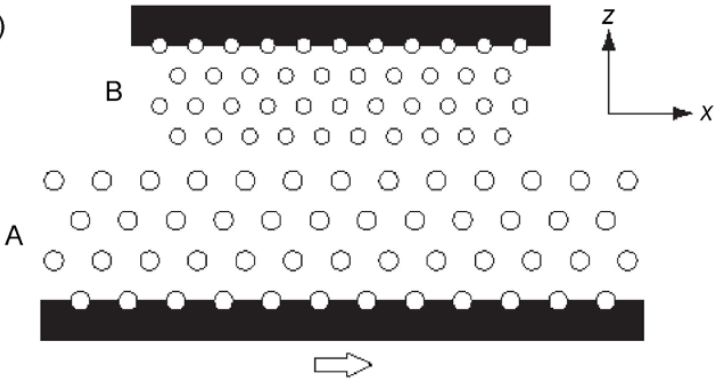

(b)

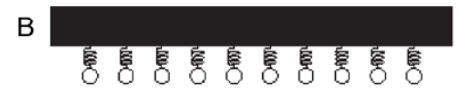

A

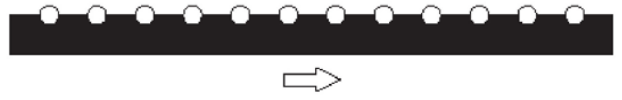

(c)

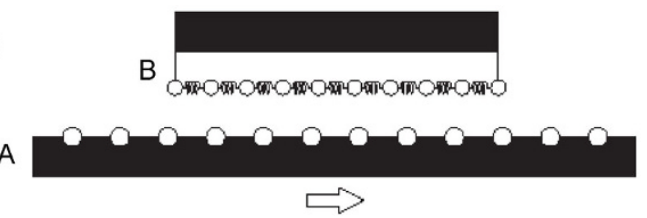

Fig. 2 Atomistic friction models. (a) Solid A sliding across solid B. (b) Independent oscillator model (Tomlinson's model). (c) FrenkelKontorova model [10].

The Frenkel-Kontorova model is a theoretical model frequently used to describe such physical systems. Sokoloff showed that the Frenkel-Kontorova model for charge density waves can reproduce phenomena such as stick-slip in friction, thus highlighting its usefulness as a model for friction in solids [12].

The idea of commensurability in solid surfaces in contact is leading to new developments in recent theoretical and experimental research in nanotribology (atomic scale friction). A sliding motion system where a one-dimensional atomic chain interacts with a periodic potential was investigated as a model of friction between ideal crystal surfaces (Frenkel-Kontorova model including the kinetic energy term) [13]. In such models of ideal crystals, the energy gain and loss of interatomic interaction energies at the sliding surface cancel each other out and the total energy of the sliding surface becomes invariant with sliding distance as long as the atomic structure of the incommensurate surface in contact is the same after atomic relaxation, therefore, the friction of an infinite system is zero at the limit of zero sliding speed [13]. In contrast, if the interaction between surfaces becomes larger than the interactions inside solids and exceeds a threshold, a structural phase transition, i.e., Aubry transition happens where locally commensurate structures appear at the incommensurate plane of contact. In this case, atoms are locally pinned, and even when the solids are adiabatically and slowly slid, the pinned atoms rapidly break bonds because of sliding, causing nonadiabatic or non-continuous motion, resulting in the dissipation of accumulated elastic energy. This is the principle of friction generation postulated by Tomlinson [5]. But how do structural phase transitions at incommensurate contact planes behave in various models? The occurrence of phase transitions is determined by the competition of interatomic interactions inside solids and between surfaces. Aubry transitions tend to appear in the one-dimensional Frenkel-Kontorova model, hence zero friction states are thought to occur only when inter-surface interactions are weak [10, 12].

In contrast, the high degree of freedom of atomic movement in models of high dimensions was pointed out to be fundamentally important in the occurrence of superlubricity, and superlubricity instead of structural phase transitions was found to occur in realistic three-dimensional systems with strong interactions such as metallic bonding [13]. In short, the concept of superlubricity, or the phenomenon of zero friction, emerged from atomistics based research on atomic scale friction $[13,14]$.

\section{Superlubricity and high dimensionality of model}

Noncontinuous motion of atoms has been demonstrated not to happen in real multiatom friction systems. To verify this, equations of noncontinuous motion were derived and the motion of atoms in real systems was evaluated to find out whether motion is continuous or not [13]. Computational experiments of a three-dimensional friction system with realistic interactions showed that adhesion several dozen times stronger than realistic adhesion is necessary for the occurrence of noncontinuous motion of atoms. Thus, individual atoms were found to undergo continuous motion is real systems, and superlubricity, or zero friction, was concluded to appear in infinite systems with incommensurate contact at the limit of zero sliding speed. Such continuous motion of atoms arises from the high dimensionality at the contact surface. Dimensionality represents the degrees of freedom of 
atomic motion at the contact surface. Figure 3 shows the stable and unstable areas of atomic motion at the contact plane. Atoms continuously move at the stable area, and atoms continue to continuously move near the unstable area because atoms can bypass around the unstable area. Such flexible motion is achieved by the high degrees of freedom of atoms. In contrast, onedimensional systems have low degrees of freedom of motion, thus any small unstable area encountered during the motion of an atom results in trapping of the atom. This atom moves noncontinuously when moving to the adjacent stable area, resulting in the generation of friction.

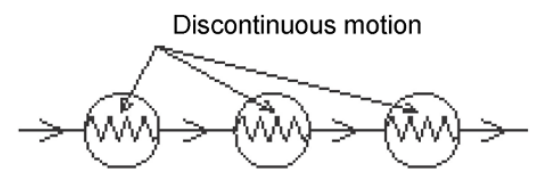

(a)

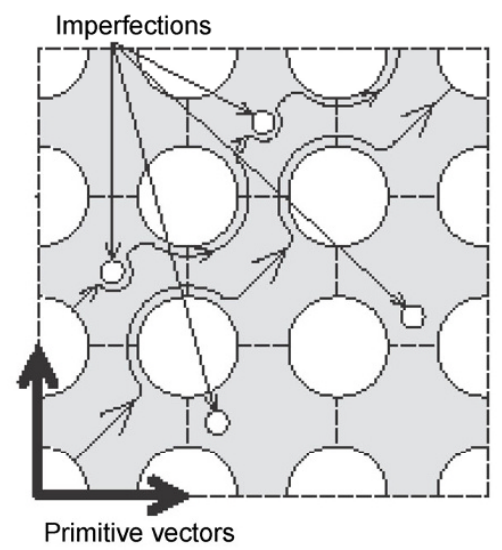

(b)

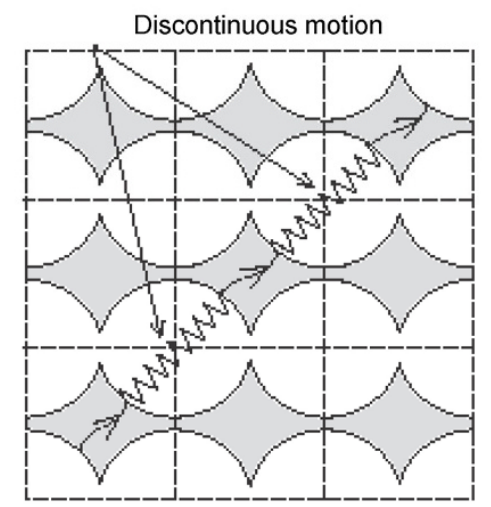

(c)

Fig. 3 Motion of atoms at contact surfaces. The white parts represent unstable areas in which atoms cannot stably exist and the shaded parts represent stable areas in which they can stably exist. (a) One-dimensional system; (b) and (c) two- and threedimensional systems.

\section{Superlubricity simulation for simple atomistic model}

\subsection{Friction model}

Figure 4 shows a one-dimensional Frenkel-Kontorova (FK) friction model. This model considers the kinetic energy of comprising atoms, and its simplicity makes it suitable for investigating the physics of friction. In the solid above, a one-dimensional chain of atoms is placed on a sinusoidal periodic potential. The atoms making up the one-dimensional chain interact with neighboring atoms through the harmonic potential of linear springs, and also experience forces from the periodic potential below and undergo sliding motion. This one-dimensional FK friction model can be extended to two- or three-dimensional models.

The Hamiltonian of the one-dimensional FK friction model can be written as

$$
\begin{aligned}
H\left(\left\{p_{i}\right\},\left\{x_{i}\right\}\right)= & \sum_{i}^{N} \frac{p_{i}^{2}}{2 m}+\sum_{i}^{N}\left\{\frac{1}{2} k\left(x_{i+1}-x_{i}-l\right)^{2}\right. \\
& \left.+\frac{f}{2 \pi} \sin \left(\frac{2 \pi x_{i}}{L}\right)\right\}
\end{aligned}
$$

where $p_{i}$ is momentum, $k$ is the spring constant, $l$ is the natural length of a spring, $L$ is the potential period of the solid below. $f$ is the amplitude of the sinusoidal potential energy, describing the adhesive interaction between the atoms of the contacting surfaces. The subsequent calculations assume $l=(1+\sqrt{5}) / 2$ and $L=1$.

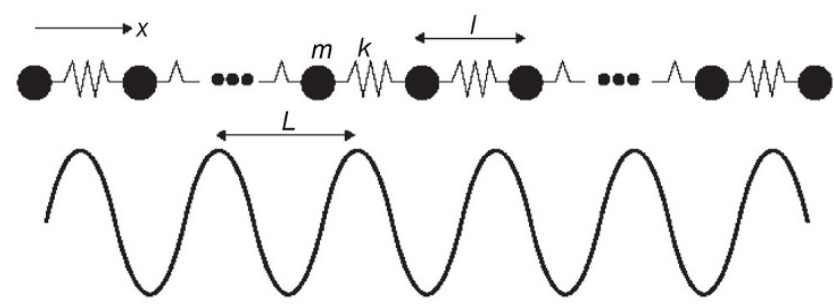

Fig. 4 Frenkel-Kontorova atomistic friction model.

\subsection{Equations of motion and dimensionless form}

The equation of motion of the one-dimensional FK friction model becomes the following from Hamilton's canonical equations: 


$$
\begin{aligned}
m \frac{\mathrm{d}^{2} x_{i}}{\mathrm{~d} t^{2}} & =-k\left\{\left(x_{i}-x_{i-1}-l\right)+\left(x_{i}-x_{i+1}+l\right)\right\}-f \cos 2 \pi x_{i} \\
& =k\left(x_{i+1}+x_{i-1}-2 x_{i}\right)-f \cos 2 \pi x_{i}
\end{aligned}
$$

where $L=1$.

This equation contains mass $m$ and a spring constant that represents interatomic interactions $k$ as parameters. These values are very small at the atomic level, and so the equation of motion is made dimensionless to ensure the accuracy of numerical calculations. The quantity $l$ in the above equation is chosen as the unit of length, and $\tau$ as the unit of time. Dimensionless quantities are denoted by an apostrophe', hence the dimensionless quantities of the coordinate component $x_{i}$ and time $t$ become $x_{i}^{\prime}$ and $t^{\prime}$, respectively. The following equation is obtained:

$$
x_{i}=l x_{i}^{\prime}, \quad t=\tau t^{\prime}
$$

Next, as,

$$
\frac{\mathrm{d}^{2} x_{i}}{\mathrm{~d} t^{2}}=\frac{\mathrm{d}}{\mathrm{d} t}\left(\frac{l}{\tau} \frac{\mathrm{d} x_{i}^{\prime}}{\mathrm{d} t^{\prime}}\right)=\frac{l}{\tau} \frac{\mathrm{d}}{\mathrm{d} t^{\prime}} \frac{\mathrm{d} x_{i}^{\prime}}{\mathrm{d} t^{\prime}} \frac{\mathrm{d} t^{\prime}}{\mathrm{d} t}=\frac{l}{\tau^{2}} \frac{\mathrm{d}^{2} x_{i}^{\prime}}{\mathrm{d} t^{\prime 2}}
$$

The equation of motion becomes the following equation:

$$
m \frac{l}{\tau^{2}} \frac{\mathrm{d}^{2} x_{i}^{\prime}}{\mathrm{d} t^{\prime 2}}=k l\left(x_{i+1}^{\prime}+x_{i-1}^{\prime}-2 x_{i}^{\prime}\right)-f \cos 2 \pi x_{i}^{\prime}
$$

The quantity $m / k \tau^{2}$ in the above equation is dimensionless, and by setting $m / k \tau^{2}=1$ we obtain:

$$
\tau=\sqrt{\frac{m}{k}}
$$

This determines the unit of time $\tau$, which is the reciprocal of the angular eigenfrequency of the onedimensional chain of atoms. An estimation of the spring constant $k$ of iron, a practical material, based on approximate calculations is $k=a E \sim 10 \mathrm{~N} / \mathrm{m}$ ( $a$ : lattice parameter, $E$ : Young's modulus), therefore $\tau$ is about 0.1 ps.

\subsection{Properties of friction and friction diagrams}

The sliding friction properties of the one-dimensional FK friction model can be investigated using molecular dynamics. In the friction simulation, the initial positions of atoms comprising the model are determined, the above solid is slid by giving an initial velocity $P(0)$ to all atoms, and the change with time regarding the positions and sliding velocities of the center of mass of solids are obtained and analyzed. Table 1 shows the calculation conditions. Here, the speed of sound of vertical waves in a solid $v$ can be written as $v=$ $\sqrt{E / \rho}=\sqrt{k / m a}$, and so can be a unit of velocity. The speed of sound in solids is around $v \sim 5,000 \mathrm{~m} / \mathrm{s}$. Therefore, if a solid slides at a constant speed of 0.1 for $10^{5}$ time, the sliding speed is around $500 \mathrm{~m} / \mathrm{s}$ and the sliding distance is about $5 \mu \mathrm{m}(=0.1 \times 5,000 \mathrm{~m} / \mathrm{s} \times$ $10^{5} \times 0.1 \mathrm{ps}$ ).

In this friction simulation, it is important to perform equilibration, where the equilibrium positions of atoms are carefully obtained, before providing initial velocities and sliding the solid above. The equilibration procedure involves obtaining a stable atomic configuration at equilibrium by adding initial velocities according to the Maxwell-Boltzmann distribution to all constituent atoms and then decreasing the temperature of the system at a sufficiently slow rate (annealing).

The friction properties of the one-dimensional FK friction model strongly depend on the intersurface interaction $f$ (adhesion), the initial velocity of the solid above $P(0)$, and the atomic configurations of the contact surfaces. An incommensurate atomic ordering at the contact surface is a necessary condition for onset of superlubricity. Therefore, the lattice parameter of the solid above is set to $l=(1+\sqrt{5}) / 2$ and that of the solid below to $L=1$. Figure 5 shows the friction properties at initial velocity $P(0)=0.2$. The friction properties indicate that the center of mass moves at constant velocity, which means that superlubricity is observed when the interatomic interaction is small at around $f=0.01$. In contrast, friction is generated and the center of mass slows down when $f$ is increased.

Table 1 Calculation conditions (values are dimensionless).

$\begin{array}{lc}\text { Atoms in model } & 10^{3} \sim 10^{4} \\ \text { Time step } \Delta t & 0.001 \\ \text { Calculation time }(=\Delta t \times \text { iteration }) & 10^{4} \sim 10^{5} \\ \text { Initial velocity } P(0) & 0.1 \sim 1.0 \\ \text { Adhesion } f & 0.01 \sim 0.14\end{array}$




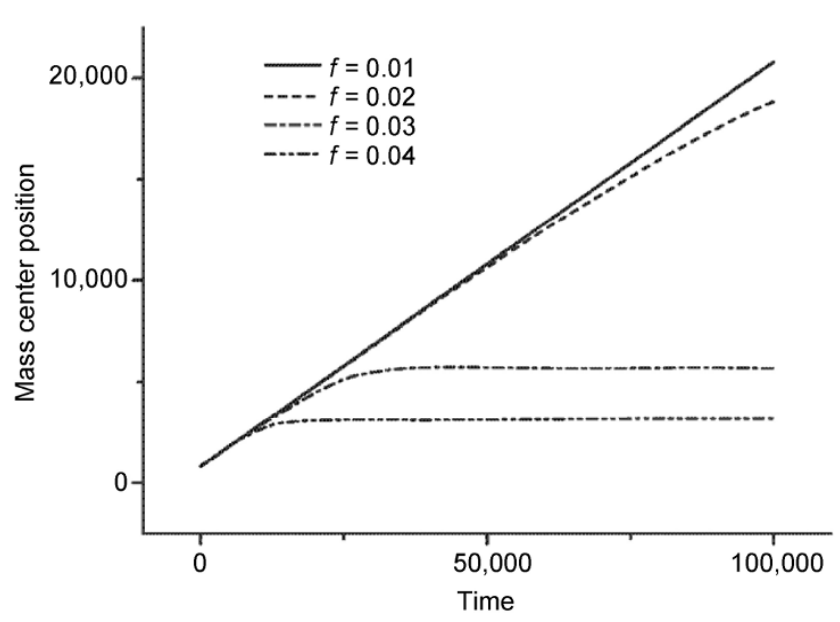

Fig. 5 Mass center position moving with time $(P(0)=0.2)$.

In this case, the translational kinetic energy of the center of mass dissipates as vibration of constituent atoms and the center of mass ultimately stops moving. Superlubricity is stable against larger intersurface interactions $f$ when the initial velocity is large.

The friction properties of the one-dimensional FK friction model depend on the interfacial interaction $f$ and initial velocity $P(0)$, which are model parameters. Two regimes, which are friction and superlubricity, are found. Obtaining friction diagrams that describe the friction and superlubricity regimes are effective for discussing friction properties. To identify the boundary between friction and superlubricity regimes, the friction force between surfaces calculated from friction simulations is evaluated and the intersurface interaction $f$ and initial velocity $P(0)$, where the friction transitions from zero to a finite value, are determined.

Figure 6 is a friction diagram derived from friction simulations with calculation time $10^{4}$. Overall, two regimes such as friction regime $(\mathrm{F})$ and superlubricity regime (S) appear. Friction regime has two sub-area of $F_{\alpha}$ and $F_{\beta}$. It seems that the boundaries between $\mathrm{F}$ area and $\mathrm{S}$ area look like irregular. It will need to perform a large number of calculations to determine more beautiful boundaries for successive study.

In this friction diagram, atoms continuously move in equilibrium positions in the area $f<0.14$ and $P(0)=0$, resulting in zero friction (superlubricity). On the other hand, the friction regime $\left(\mathrm{F}_{\alpha}\right)$ extends if $0<f<0.14$ and the initial velocity is finite $(P(0) \neq 0)$. In summary, superlubricity is likely to happen when the intersurface

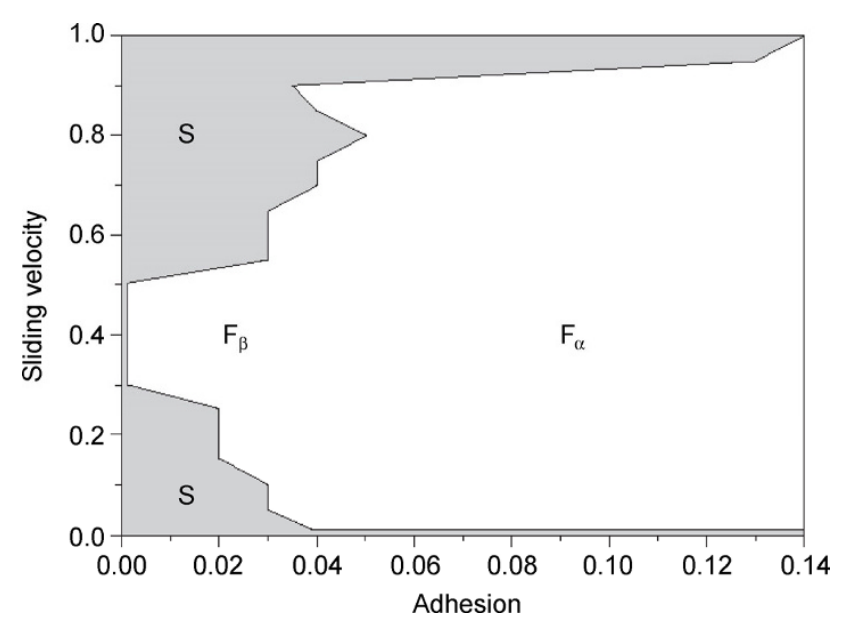

Fig. 6 Friction phase diagram of Frenkel-Kontorova model.

interaction parameter is small because of dynamic effects of kinetic energy. Interestingly, superlubricity is specifically unlikely to appear at the range of intermediate velocity $(0.3-0.5)$ even if the intersurface interaction is small, and a friction regime with different friction properties $\left(\mathrm{F}_{\beta}\right)$.

The friction system of multi particles, which are coupled with each other while feeling the sinusoidal potential when mass center moves, is essentially nonlinear dynamical system. In superlubricity case, it is easier to evaluate the vibration mode which looks like simple harmonic oscillation mode, where a primary vibration peak and the associated higher order peaks have been verified.

When sliding speed approaches to the region of $\mathrm{F}_{\beta}$ area at small $f$, simple almost harmonic mode turns to be non-harmonic mode when multi vibration peaks have appeared. The mechanism for the appearance of the multi peaks is complex problem; it will need to elaborately examine vibration mode signals in terms of non-linear system analysis. A scenario for the transition superlubricity $(S)$ mode to Friction $\left(F_{\beta}\right)$ could be described in terms of several non-linear vibrations such as subharmonic oscillation, summedand-differential harmonic oscillation, superharmonic oscillation, and internal resonance phenomena.

In the case of $F_{\alpha}$ mode, the dynamics of the system has shown to be chaotic. The occurrence of irreversible energy dissipation indicates that the internal relative motions are excited, and hence the adiabaticity does not hold true for the internal relative motions. In order that the present idea is adequate, the system must 
work as a host system absorbing energy. In other words, the dynamic system has the ergodic property. Our simulations showed that, for the frictional systems of the current interest, the energy seems to be distributed over the entire degrees of freedoms in the case of $\mathrm{F}_{\alpha}$ mode [13].

\section{Atomistic simulation for realistic systems}

\subsection{Friction transition}

The simple friction models in the previous chapter are suitable for discussing the physics of friction. However, we want to know whether superlubricity can stably exist in real systems with more complex interatomic interactions. The total energy in incommensurate contact surfaces can be invariant with sliding distance $Q$ in some situations, resulting in vanishing friction. When the interfacial interaction (adhesion) exceeds a certain threshold, a structural transition happens and commensurate structures are found locally in the incommensurate contact surface. Atoms then become pinned locally, energy dissipates, and friction arises. Therefore, a "friction transition" happens [13], where friction becomes finite from zero, with increasing adhesion at incommensurate surfaces.

Superlubricity is not observed when there is a friction transition at an incommensurate contact surface. Real systems with metallic bonding were modeled using a Morse pair potential to investigate friction transitions in a metallic system [13]. The criterion that determines the occurrence of the friction transition was obtained. This criterion judges whether atoms on the sliding surface can keep their equilibrium positions; superlubricity becomes unstable and friction arises if the equilibrium positions cannot be maintained. The criterion evaluates the sign of the second derivative of the potential energy of the atom of interest $V(Q, r)$ at a given position, that is, the sign of $\mathrm{d}^{2} V(Q, r) / \mathrm{d} r^{2}$. It is important to obtain the correct equilibrium position of the atom in this judgment, thus the derivation of interatomic potentials that can be applied to materials of interest is critical. Research on metallic systems where the Morse potential can be used showed that friction transitions do not happen under strong metallic bonding interactions [13].
Atomic-level friction experiments were carried out using scanning tunneling microscopy based on this conclusion [13]. The (011) surface of a clean tungsten tip needle and the (001) surface of a single crystal of silicon were used. An ultrafine structure friction system at the atomic scale was constructed with these surfaces, and the friction between the clean surfaces under elastic contact was measured while controlling the distance between the contact surfaces to close to the interatomic distance. The friction at the incommensurate contact surface of tungsten and silicon was below the measurement resolution and so could not be detected. This experimental result shows that there was no friction transition in this experimental system with incommensurate contact surfaces. A theoretical analysis of the friction transition in a tungsten and silicon friction system is necessary to understand the theoretical reasons behind these results (Fig. 7).

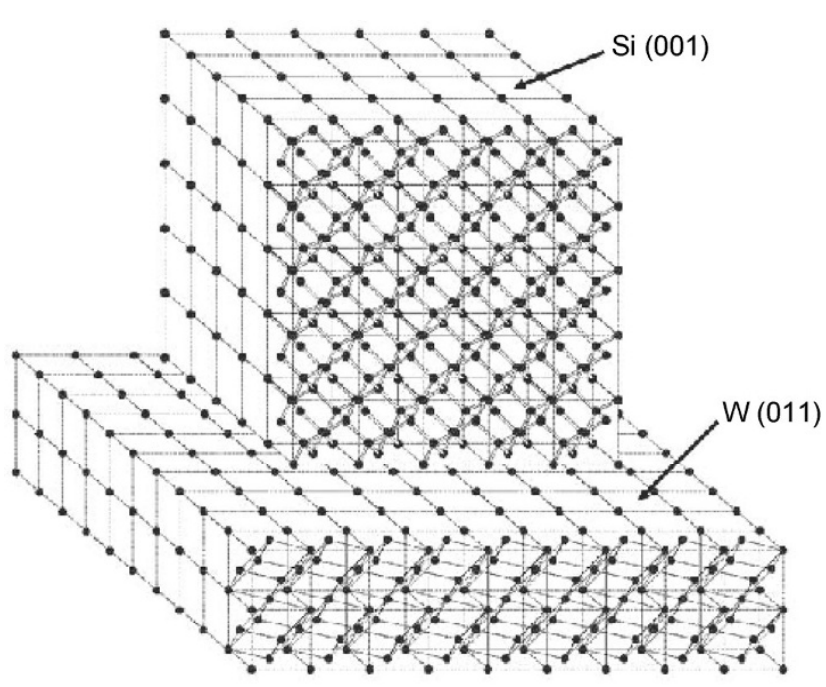

Fig. 7 Atomistic model of Si (001) and W (011) for evaluating friction transition.

\subsection{Many-body potentials in real systems}

A reliable interatomic potential is necessary to investigate friction transitions in a real tungsten-silicon system. Two-body (central force) potentials are useful in metals with simple electronic structures; however, three-body potentials that take the local environment of interatomic interactions into consideration need to be constructed for each system to be researched that contains complex electronic structures, which include transition metals and semiconductors. 
Various three-body potentials have been proposed for various materials and materials properties that are investigated. In a friction system containing tungsten (W) and silicon (Si), interatomic potentials for $\mathrm{W}-\mathrm{Si}$, $\mathrm{Si}$, and $\mathrm{W}$ are necessary. A quantitatively accurate potential with a small number of parameters [15] was adopted as a realistic potential that models friction. This potential has the form

$$
V_{i j}=A \exp \left[-\beta\left(r_{i j}-R_{i}\right)^{\gamma}\right]\left[\exp \left(-\theta r_{i j}\right)-\frac{B_{0}}{Z_{i}^{\alpha}} \exp \left(-\lambda r_{i j}\right) G(\eta)\right]
$$

where $r_{i j}$ is the interatomic distance and $R_{i}$ is the distance between nearest neighbor atoms. This potential contains the eight parameters $A, B_{0}\left(=B C^{\varepsilon}\right)$, $\theta, \lambda, \alpha, \beta, \gamma, \eta$.

$G(\eta)$ represents three-body effects arising from the differences in local environments of interatomic interactions, and is given by the following equation that considers local atomic configurations:

$$
G(\eta)=1+\sum_{k \neq i, j}\left[\cos \left(\eta \Delta \theta_{j i k}\right)-1\right]
$$

where $\eta$ is the bond angle parameter, and $\Delta \theta_{j i k}$ is the difference between the interatomic bond angle between atoms of interest $\theta_{i j k}$ and the equilibrium bond angle $\theta_{i}\left(109.47^{\circ}\right.$ in the diamond structure), or

$$
\Delta \theta_{j i k}=\left|\theta_{j i k}-\theta_{i}\right|
$$

To obtain the potential parameters above, the following expression for cohesive energy $E_{\mathrm{b}}$ that describes universal properties of cohesion of various materials is used.

$$
E_{\mathrm{b}}=Z A\left[\exp (-\theta r)-B p^{\varepsilon} \exp (-\lambda r)\right]
$$

where $Z$ is the coordination number, $p\left(p^{\varepsilon}=C^{\varepsilon} / Z^{\alpha}\right)$ is the bond order, and $A, B, \theta, \lambda$ are potential parameters (previously defined). The cohesive energy is given in the form of pair potentials plus a three-body correction term that depends on the local environment. The equilibrium interatomic distance $r_{\mathrm{e}}$ satisfies $\mathrm{d} E_{\mathrm{b}}$ $/ \mathrm{d} r=0$ and is given by

$$
r_{\mathrm{e}}=\frac{1}{\theta-\lambda} \ln \left(\frac{S}{B p^{\varepsilon}}\right)=\frac{1}{\theta-\lambda} \ln \left(\frac{S Z^{\alpha}}{B_{0} C^{\varepsilon}}\right)
$$

where $S=\theta / \lambda, \alpha=\delta \varepsilon$ and $p=C / Z^{\delta}$ are assumed.

The cohesive energy at the equilibrium interatomic distance $r_{\mathrm{e}}$ is

$$
D_{\mathrm{e}}=Z A(S-1) \exp \left(-\theta r_{\mathrm{e}}\right)=Z A B p^{\varepsilon} \frac{S-1}{S} \exp \left(-\lambda r_{\mathrm{e}}\right)
$$

The above relations show that the equilibrium interatomic distance $r_{\mathrm{e}}$ is proportional to $\ln \left(D_{\mathrm{e}} / Z\right)$ and $\ln (Z)$. Figure 8 shows the relation between $r_{\mathrm{e}}$ $\ln \left(D_{\mathrm{e}} / Z\right)$ and $r_{\mathrm{e}}-\ln (Z)$ for various crystal structures of silicon. Table 2 shows the potential parameters of $\mathrm{Si}$, $\mathrm{W}$, and $\mathrm{W}-\mathrm{Si}$ derived using the above method [16].

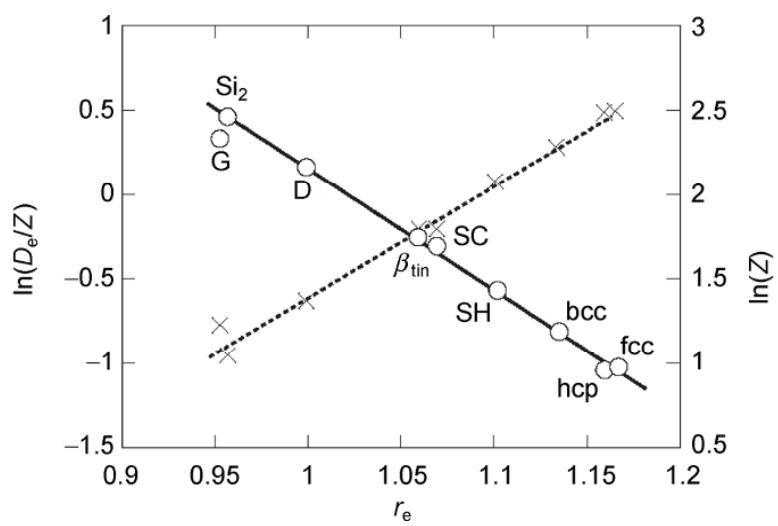

Fig. 8 Plots of $\ln \left(D_{\mathrm{e}} / Z\right)$ (solid line) and $\ln (Z)$ vs. $r_{\mathrm{e}}$ for a range of structure of silicon, where $\mathrm{G}$ and $\mathrm{D}$ represent benzene and diamond.

Table 2 Potential parameters of Si, W, and W-Si.

\begin{tabular}{cccc}
\hline Parameters & $\mathrm{Si}$ & $\mathrm{W}$ & $\mathrm{W}-\mathrm{Si}$ \\
\hline$A$ & 2794.2386 & 3798.6189 & 38444.1675 \\
$B_{0}$ & 0.08251716 & 0.251094 & 0.169645 \\
$\theta$ & 3.13269 & 2.68935 & 2.79434 \\
$\lambda$ & 1.34146 & 1.52280 & 1.70172 \\
$\alpha$ & 0.6249096 & 0.548241 & 0.285641 \\
$\beta$ & 25.44123 & 20.75723 & 25.42105 \\
$\gamma$ & 3.38218 & 3.26327 & 3.39927 \\
$\eta$ & 0.90084597 & 0 & 0 \\
\hline
\end{tabular}

\subsection{Stability analysis of superlubricity}

The friction transition in a silicon-tungsten friction model was investigated using an interatomic potential that considers many-body effects described by the potential parameters in Table 2 [16]. Based on the atomistic model in Fig. 7, the Si (001) surface is placed 
in contact with the $W(011)$ surface at a lattice misfit angle $\Theta$ as in Fig. 9. The solid above is a $5 \times 5 \times 5$ diamond structure crystal (1,625 atoms), and the solid below is an $18 \times 18 \times 2$ face-centered cubic lattice $(3,423$ atoms). Atoms in the solid below are fixed, and the conditional expression of friction transition was evaluated by obtaining the equilibrium positions of atoms in the above solid. Here, the W (011) surface of tungsten, which is the densest surface (hardest surface), was used. Investigation of the conditional expression of friction transition showed that friction transitions do not happen at the silicon-tungsten incommensurate contact surface under contact conditions for superlubricity. In other words, superlubricity was found to exist and is stable in this incommensurate contact surface.
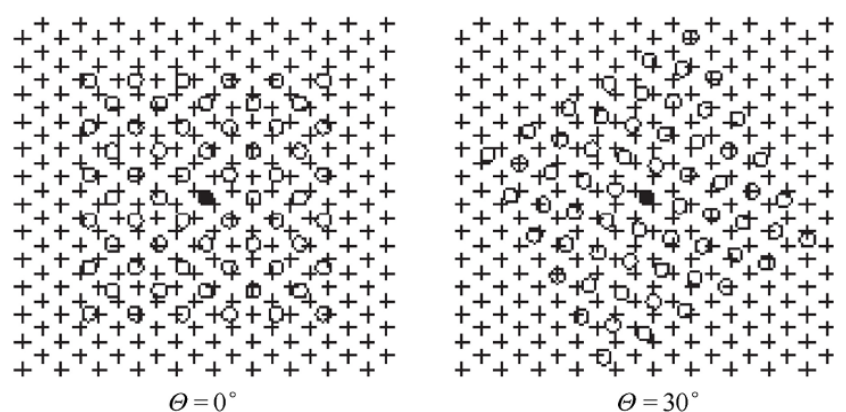

Fig. 9 Interface model for friction transition in realistic systems of Si (001) and W (011).

\subsection{Realistic macro scale superlubricity}

More realistic case such as superlow friction material has been investigated. The ultra-low friction coefficient of Molybdenum disulfide $\left(\mathrm{MoS}_{2}\right)$ film observed on a macroscale has been explained as originating in the observed atomic structure of incommensurate intrasolid sliding planes [17]. $\mathrm{MoS}_{2}$ is a well-known lamellar solid lubricant with a hexagonal structure. The low friction coefficients between 0.01 and 0.05 have been measured and the lowest one of magnitude of $10^{-3}$ range has been found when testing a sputtered $\mathrm{MoS}_{2}$ coating exempt of impurities such as carbon, oxygen, and water vapor using ultrahigh vacuum analytical tribotester. A friction coefficient in the $10^{-3}$ range is associated with friction-induced orientation of "easy shear" basal planes of the $\mathrm{MoS}_{2}$ crystal structure parallel to the sliding direction. In addition to this basal plane orientation, an orientation disorder around $c$ axis is observed by using transmission electron microscope, showing frictional anisotropy stemmed from commensurability during intercrystallite slip could be the origin of the superlow friction coefficient [17]. Recently molecular dynamics calculations have shown the superlubricity of $\mathrm{MoS}_{2}$ under vacuum [18]. Another case of superlow friction is hydrogenated diamond-like carbon (DLC) coating film sliding also in ultrahigh vacuum conditions [19]. The computer simulation studies have investigated the mechanisms of the superlow friction of DLC film, hopefully leading to presenting the guideline for synthesizing DLC with high quality.

\section{Summary}

The finding that superlubricity can exist in real systems arises from the theoretical study on the atomistic origin of friction, which considers that matter is composed of atoms, and was discovered during research on friction based on atomistics. The problem of the origin of friction has attracted great interest throughout the history of friction research in relation to the problem of friction heat in thermodynamics. The irreversibility of heat phenomena such as friction heat can be handled by introducing friction and viscosity to the equations of motion. However, it is necessary to investigate the structure of materials to explain the causes of friction and viscosity. As a result, friction research at the atomistic level has become a new area of nanotribology research.

The results of research on applications of superlubricity [20] are likely to lead to a revolution in engineering technology in relation to a sustainable society and energy problems.

Open Access: This article is distributed under the terms of the Creative Commons Attribution License which permits any use, distribution, and reproduction in any medium, provided the original author(s) and source are credited.

\section{References}

[1] Sota N. Masatsu no Hanashi (in Japanese). Tokyo: Iwanami Shoten, 1980. 
[2] Hardy W B, Hardy J K. Note on static friction and on the lubricating properties of certain chemical substances. Phil Mag 38: 32-40 (1920)

[3] Holm R. Electric Contacts. Berlin: Spring Verlag, 1967.

[4] Tabor D. Friction-The present state of our understanding. Trans ASME, J Lub Technol 103: 169-179 (1981)

[5] Tomlinson G A. A molecular theory of friction. Phil Mag 7: 905-939 (1929)

[6] Krim J. Friction at the atomic scale. Sci Am 275: 74-80 (1996)

[7] Bhushan B, Isralachvili J N, Landman U. Nanotribology: Friction, wear and the atomic scale. Nature 374: 607-616 (1994)

[8] Thompson P A, Robbind M O. Origin of stick-slip motion in boundary lubrication. Science 250: 792-794 (1990)

[9] Mate C M, McClelland G M, Erlandsson R, Chiang S. Atomic-scale friction of a tungsten tip on a graphite surface. Phys Rev Lett 59: 1942-1945 (1987)

[10] McClelland G M. In Friction at Weakly Interacting Interfaces, Adhesion and Friction. Grunze M and Kreuzer H J Ed. Berlin: Springer, 1990: 1-16.

[11] Bak P. Commensurate phases, incommensurate phase, and the devil's staircase. Rep Prog Phys 45: 587-629 (1982)

[12] Sokoloff J B. Theory of dynamical friction between idealized sliding surfaces. Surf Sci 144: 267-272 (1984)

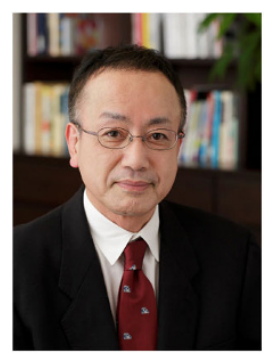

Motohisa HIRANO. He rseceived bachelor and master degrees of the Mechanical Engineering of Nagoya University. He received $\mathrm{PhD}$ degree of Engineering from Nagoya University in 1989 and PhD degree of Science from The University of Tokyo in 1998. He joined the Nippon
[13] Hirano M. Atomistics of friction. Surf Sci Rep 60: 159-201 (2006)

[14] Erdemir A, Martin J-M. Superlubricity. Oxford (UK): Elsevier, 2007.

[15] Khor K E, Das Sarma S. Proposed universal interatomic potential for elemental tetrahedrally bonded semiconductors. Phys Rev B 38: 3318-3322 (1988)

[16] Hirano M, Murase H, Nitta T, Ito T. Evaluation of friction transition for metal-semiconductor interfaces using model potential comprising three-body contributions. J Phys: Conf Ser 258: 012014 (2010)

[17] Martin J M, Donnet C, Le Mogne T, Epicier T. Superlubricaty of molybdenum disulphide. Phys Rev B 48: 10583 10586 (1993)

[18] Onodera T, Morita Y, Suzuki A, Koyama M, Tsuboi H, Hatakeyama N, Endou A, Takaba H, Kubo M, Dassenoy F, et al. A computational chemistry study on friction of h-MoS Part I: Mechanism of single sheet lubrication. J Phys Chem B 113: 16526-16536 (2009)

[19] Fontaine J, Donnet C, Grill A, LeMogne T. Tribochemistry between hydrogen and diamond-like carbon films. Surf Coat Tech 146-147: 286-291 (2001)

[20] Liu Z, Yang J, Grey F, Liu J Z, Liu Y, Wang Y, Yang Y, Cheng Y, Zheng Q I. Observation of microscale superlubricity in graphite. Phys Rev Lett 108: 205503 (2012)

Telegraph and Telephone Corporation from 1982 to 2003. He moved to Gifu University in 2003 and was a Professor of the Department of Mathematical and Design Engineering Department from 2003 to 2014. He then moved to Hosei University in 2014 and his current position is a Professor at the Department of Mechanical Engineering of the Faculty of Science and Engineering of Hosei University. 